\title{
Sensing and Control Electronics for a Benchtop Hybrid Powertrain
}

\section{Dr. Eric Constans, Rowan University}

Eric Constans is an Associate Professor in Mechanical Engineering at Rowan University. His research interests include engineering education, mechanical design and acoustics and vibration.

\section{Mr. Karl Dyer, Rowan University}

Karl Dyer is a Mechanical Engineering Technician at Rowan University. He received his B.S. in mechanical engineering and M.S. in electrical engineering from Rowan University. 


\title{
Sensing and Control Electronics for a Benchtop Hybrid Powertrain
}

\begin{abstract}
Concept retention between courses is a recurring problem for engineering educators - one that is exacerbated by the disjointed nature of the engineering curriculum. One possible solution to the problem, a multi-year design/build/test project, is currently being studied by the authors. The project, a bench-scale hybrid powertrain, is completed by our students over the course of five semesters. The focus of this paper is the set of electronic circuitry needed to sense and control the powertrain. This is the latest installment in a series of papers discussing the project; see [2-6] for a fuller description.

The "prime mover" in the benchtop hybrid is a small engine powered by compressed air, which is designed and fabricated by students during their Junior year. The goal of the powertrain is to convert the maximum amount of energy stored in the compressed air to motion at the output shaft of the powertrain. In order to accomplish this, the students need a means of sensing the speeds of each of the shafts in the powertrain, as well as monitoring the amount of compressed air being sent to the engine and the electrical power sent to/from the battery pack. In addition, the instructor needs to measure compressed air use and to provide a controlled load at the output of the powertrain. Some parts of the sensing/control circuitry (e.g. the tachometer) are built by the students, while other parts are fabricated by the faculty as part of the set of benchtop "workstations" used by the students. This paper will present and discuss each of the electronic circuits in the sensing/control/loading system.
\end{abstract}

The circuits described in the paper (e.g. tachometer, motor driver, electrical load) have wide application in automotive engineering and robotics, and it is hoped that by presenting a thorough description of each circuit, instructors at other institutions can benefit from our successes and failures and adopt individual modules from the hybrid powertrain into their own laboratory instruction.

\section{Introduction}

One of the most exciting innovations in automotive technology is the development and realization of the hybrid-electric powertrain. The most commercially successful hybrid vehicle has been the Toyota Prius [1]. Students at Rowan University design and fabricate a bench-scale version of the Toyota Hybrid System over the course of five semesters, as a way of integrating the mechanical engineering curriculum. Parts of this project have been described in earlier papers [2], [3], [4], [5] and only a short background will be given here. A wealth of information about the project is available on the author's website www.benchtophybrid.com.

It is important to note that we are mechanical engineers and our areas of expertise do not cover electronic circuit design. While we have frequently consulted with our colleagues in the Electrical Engineering department, much of what is discussed in this paper has been learned through trial and error in conducting this project with our students. This paper discusses a set of experiments that faculty members at other institutions who wish to devise hands-on projects for their students in robotics, controls, or other electromechanical systems. The circuits described 
here have wide application, for example the motor/generator board could easily be adapted for use in a benchtop dynamometer, and the inexpensive, reliable tachometer can be incorporated into any speed control system. Of course, the authors welcome any suggestions for improvement to the circuits described here.

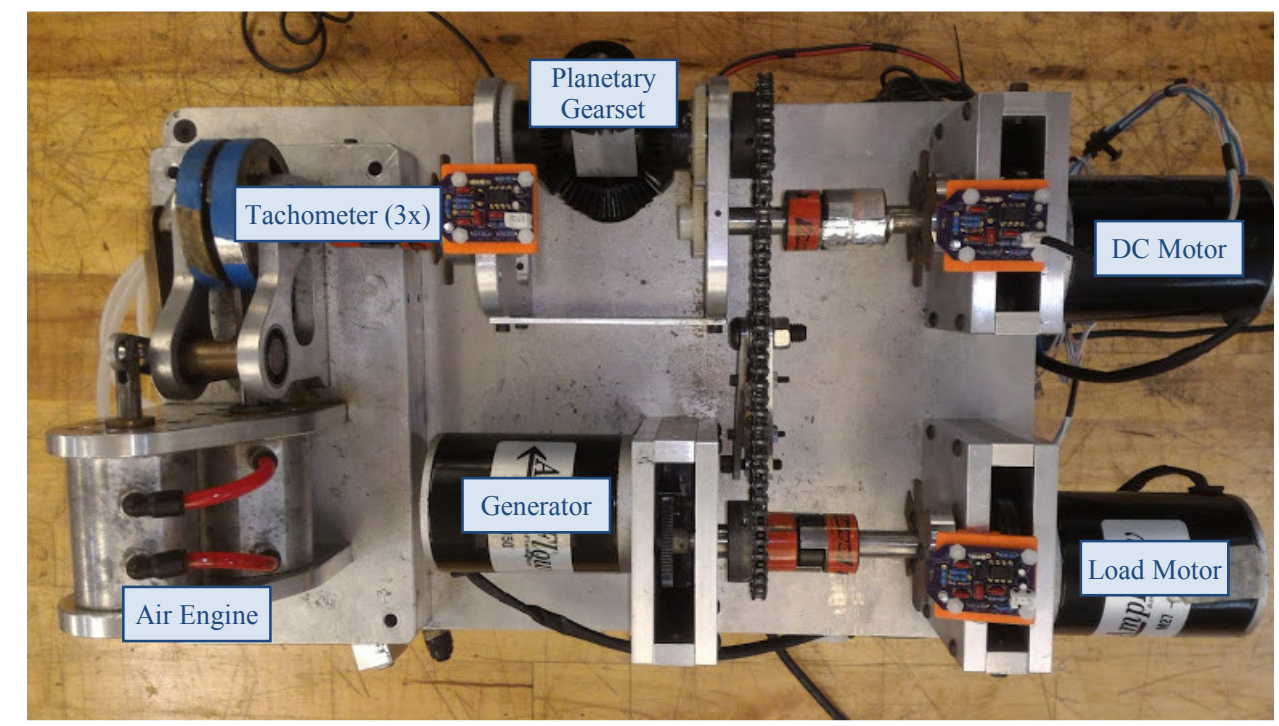

Figure 1: Overhead view of benchtop hybrid powertrain. All three motor/generators are identical, but have differing roles in the powertrain. The three tachometer boards are used to sense rotational speeds at important points in the powertrain.

\section{The Benchtop Hybrid}

Figure 1 shows an overhead view of the bench-scale hybrid powertrain that students in our program work with. The main difference between the bench-scale version and the Toyota Hybrid System is that an air engine is used instead of a gasoline-powered engine as the prime mover [6]. Driving torque is provided by the Air Engine and DC motor, and the Generator is used to store energy during coasting or downhill portions of the driving cycle. The Load Motor is used by the instructor to provide a driving cycle for the powertrain. During an uphill stretch the Load Motor is used as a generator, which puts a load on the powertrain. During downhills, the Load Motor is driven as a motor, which "back drives" the powertrain, allowing energy to be stored by the Generator. There are three Tachometers, which measure the speed of each shaft in the powertrain. The top two tachometers are used by the students to control the speed of the Air Engine and DC Motor and the lower tachometer, mounted to the load motor, is used by the instructor to measure the deviation from set point speed of the powertrain.

There are three major electronic components in the powertrain that will be discussed in this paper. The Tachometer Board is used to measure the rotational speed of a particular shaft in the powertrain. The Motor/Generator Board is used to drive a DC motor or to provide an electrical load when it is used as a generator. And the Solenoid Board is used to regulate the speed of the Air Engine. All of these circuits have been designed and tested by us, and have been used by students in completing the Benchtop Hybrid project. The full circuit diagrams and EAGLE CAD files can be found on the Benchtop Hybrid website, www.benchtophybrid.com. 


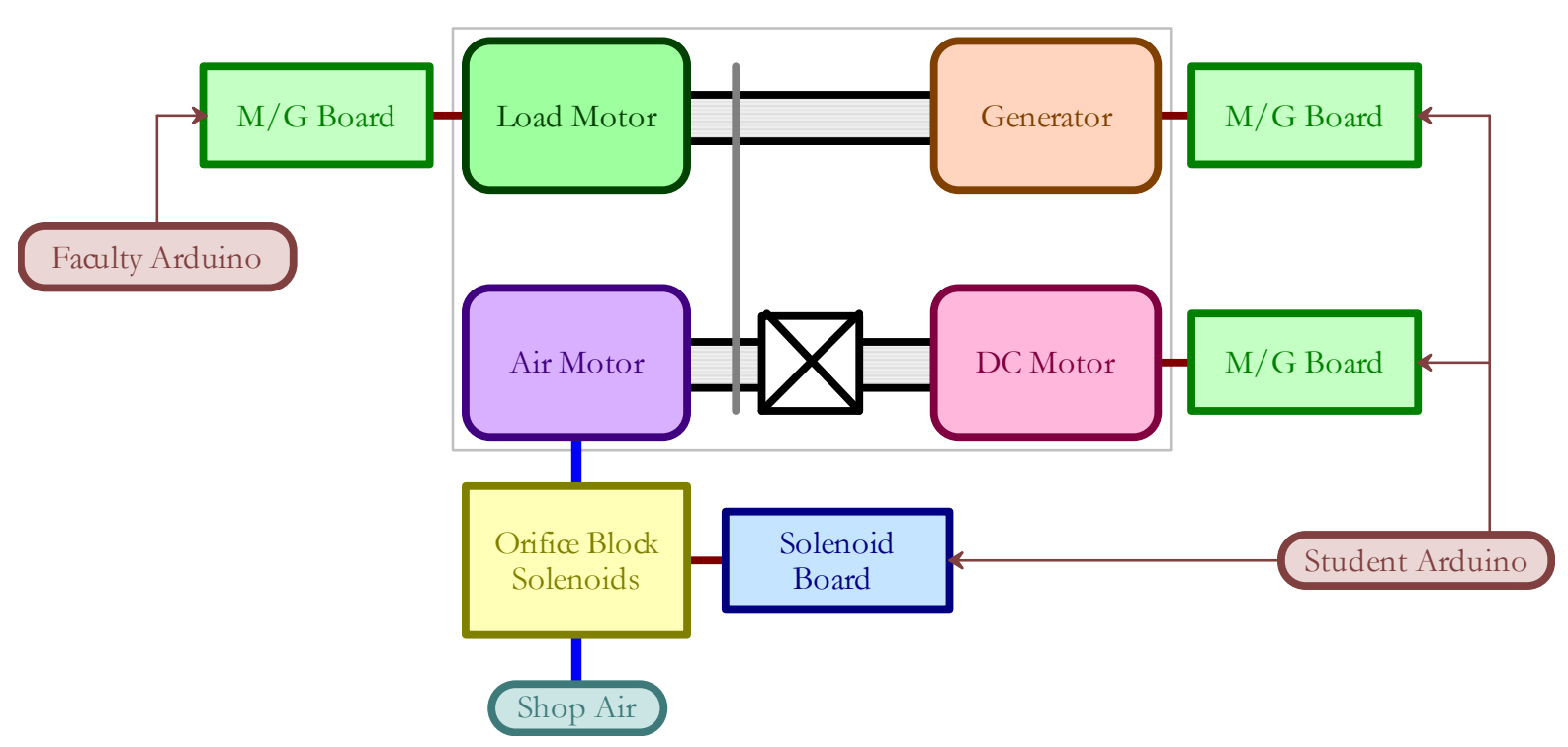

Figure 2: Control diagram of benchtop hybrid powertrain. The items discussed in this paper include the Motor/Generator Board, The Solenoid Board and the Tachometer Board.

A control diagram of the Benchtop Hybrid system is shown in Figure 2 above. The Motor/Generator Board was designed as a general-purpose system, capable of driving a DC motor or providing an electrical load. The Solenoid Board controls the solenoid valves that regulate the speed of the Air Engine. A Tachometer Board (not shown) is used to measure the speed of each shaft in the system. To achieve control of the system, a student must measure the input shaft speeds (using the Tachometer Board) of the Air Engine and DC motor, monitor the current supplied to the DC motor and the charge recuperated using the generator, and monitor the amount of air supplied to the Air Engine via the Solenoid Board. The instructor must monitor the same quantities, as well as the speed at the output shaft to ensure that the set point speed is achieved.

In early versions of the Benchtop Hybrid, we required the students to use the generator to store energy in a battery pack; this energy would be used later to complete other parts of the drive cycle. In practice, we found that this had two major disadvantages in a student environment:

1. During testing of the overall control scheme, it was difficult to ensure that each team would start with a completely discharged battery. Therefore, it was difficult to achieve an "even playing field" when assessing each team's performance relative to the class average.

2. The storage capacity of each battery pack varied with age, and with previous charge/discharge cycles. The "even playing field" problem obtained here as well.

To eliminate these problems, we allowed students to store energy in a "virtual battery". The instructor and students monitored the current flow from the generator, and integrated it to calculate a total "state of charge". The current flowing to the DC motor was also monitored, and the instructor calculated the following quantity in real time: 


$$
S_{C}=\int_{0}^{t} i_{g} d t-\int_{0}^{t} i_{m} d t
$$

where $i_{g}$ is the current flowing from the generator and $i_{m}$ is the current flowing to the DC motor. The state of charge, $S_{C}$, must be positive at the end of the drive cycle, or the student team received a penalty. We explained to the students that this equation ignores efficiencies in battery storage and discharge, but we could easily build in correction factors for these effects in later versions.

Of the circuits described in this paper, the students were responsible for analyzing and fabricating only the Tachometer Board. The Motor/Generator and Solenoid Boards were provided to the students as part of each benchtop workstation; the students use their Arduino boards to interface with and control these circuits.

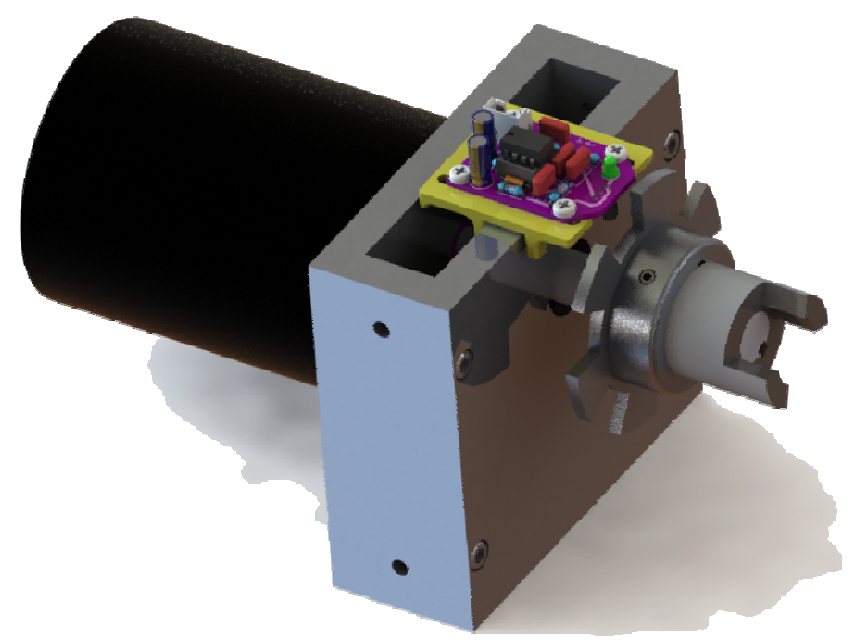

Figure 3: The tachometer board (purple) shown attached to the output of a DC motor. The daisywheel is the disk with six "petals" shown in front of the tachometer and the Hall-Effect sensor is the small black object hanging below the board.

\section{The Tachometer Board}

The purpose of the Tachometer Board, shown in Figure 3, is to enable a student's Arduino microcontroller to measure the speed of a rotating shaft. To accomplish this, a "daisywheel" (a disk with slots around the periphery) is mounted on the shaft, and a fixed sensor (optical or magnetic) is used to count the pulses as the daisywheel rotates past. Over the past five years, we have designed and tested several types of tachometer, with varying degrees of success. Our first attempt used optical sensors to count pulses, but these were found to be highly susceptible to interference from outside light sources. Students would calibrate their tachometers in our windowless computer lab, and then obtain completely different results when they brought their system into our sunlit Projects Laboratory. Thus, the optical sensor tachometer was abandoned after the first year. 
Our next attempt used a Hall-Effect sensor to detect pulses from the daisywheel. This has the advantage of not being susceptible to light pollution, but requires that the daisywheel be made of a ferrous material. Our students have access to an abrasive waterjet cutter, so this presents no difficulty at our institution; students lacking access to this type of machine will need to find an alternate method of fabricating a ferrous daisywheel.

Our early tachometer designs required a student to count the daisywheel pulses using the interrupt function on the Arduino. Dividing the number of pulses by the elapsed time gave the rotational speed of the shaft. Unfortunately, this "digital" tachometer was found to be quite error-prone, owing largely to the sometimes unpredictable behavior of the Arduino interrupt function. While the faculty prototype of this design functioned with reasonable robustness, we soon found ourselves spending an inordinate amount of time debugging student code. The Arduino-based digital tachometer was found to be too unreliable for use in a speed control system. Thus, we have chosen a Hall-Effect analog tachometer as our final design.

On the simplest level, the digital tachometer circuit takes magnetic pulses in at one end, and generates a voltage (to be measured with an Arduino analog port) at the other end. The voltage is proportional to the speed at which the magnetic pulses arrive at the input.

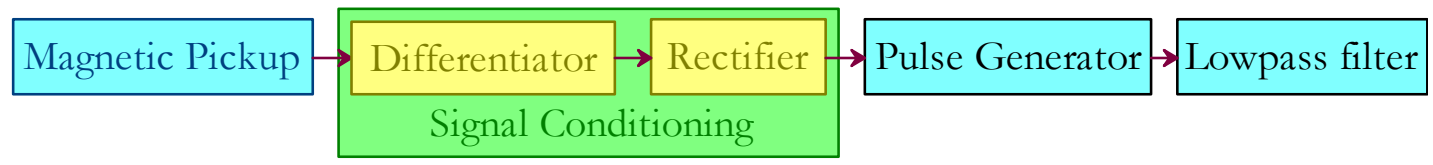

Figure 4: Functional diagram of the tachometer circuit. The heart of the circuit is a Hall-Effect sensor, the Melexis MLX90217. The rest of the circuit converts pulses from the daisywheel to an analog voltage signal.

Figure 4 gives a functional diagram of the circuit. At the beginning, there is a magnetic pickup that detects the presence of a ferrous material and generates a pulse. The signal conditioning circuitry changes the pulse into a trigger that activates the pulse generator. When the pulse generator receives a trigger, it creates a pulse of an exact duration. The lowpass filter determines the average voltage that it sees - the more pulses it sees per second, the higher the average voltage. The filter sends this average value to the Arduino, where it can be translated into a rotational speed.

\section{Magnetic Pickup}

At the heart of the magnetic pickup circuit is the Melexis MLX90217 Hall Effect Geartooth Sensor. Figure 5 shows a diagram of the Hall-Effect sensor circuit. An LED is used as a visual check that the power is on and the sensor is functioning correctly. Thus, the magnetic pickup circuit generates $0 \mathrm{~V}$ when it detects the daisywheel petal, and 5V when it doesn't. The result is a regular sequence of pulses whose frequency depends upon the rotational speed of the daisywheel.

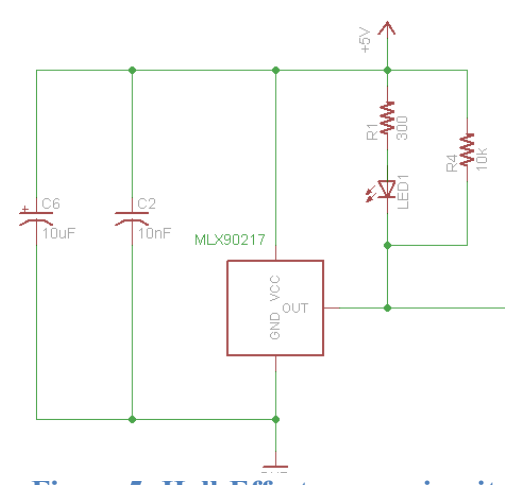

Figure 5: Hall-Effect sensor circuit. 


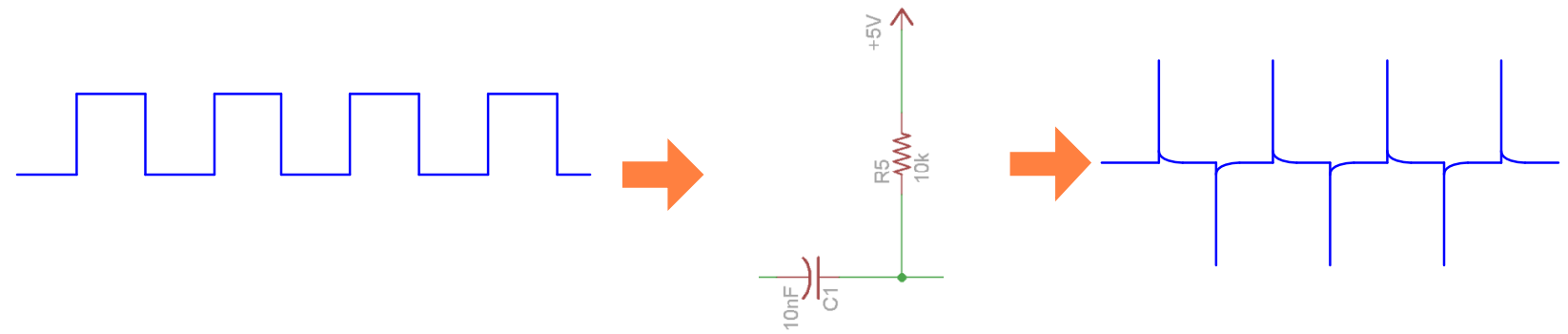

Figure 6: The differentiator converts a square wave into a series of trigger pulses.

\section{The Differentiator}

The next few modules in the circuit transform the square wave into something that the pulse generator can recognize. First, note that the pulse generator can only be triggered by a negative pulse of very short duration. If a longer pulse is used to trigger the pulse generator, multiple output pulses may result for a single input pulse. One simple way to create pulses is to differentiate the square wave. The result of differentiating the square wave is a series of positive and negative "spikes", as shown in Figure 6. A differentiator can be constructed using a highpass filter, as shown in the circuit in the figure.

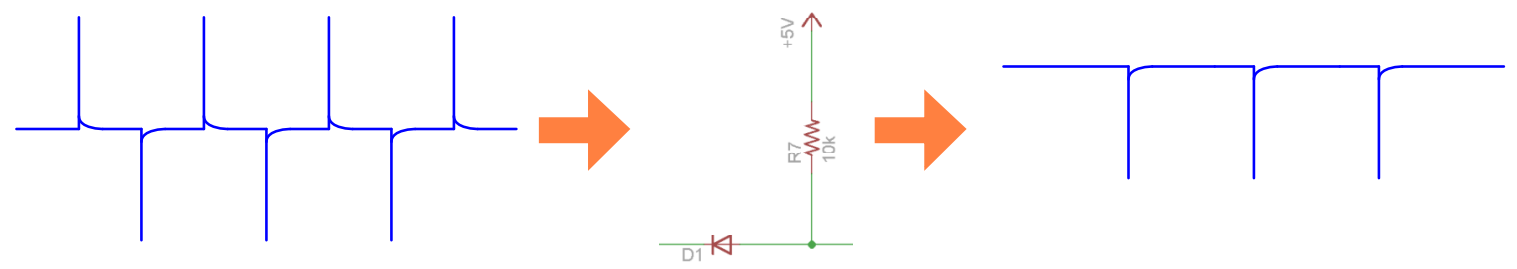

Figure 7: The rectifier removes positive pulses and allows only negative pulses to pass through.

\section{The Rectifier}

The pulse generator is triggered by negative pulses, so the purpose of the rectifier is to eliminate the positive pulses, allowing only the negative pulses to pass through. As shown in Figure 7, the diode blocks positive pulses, and allows negative pulses to pass through.

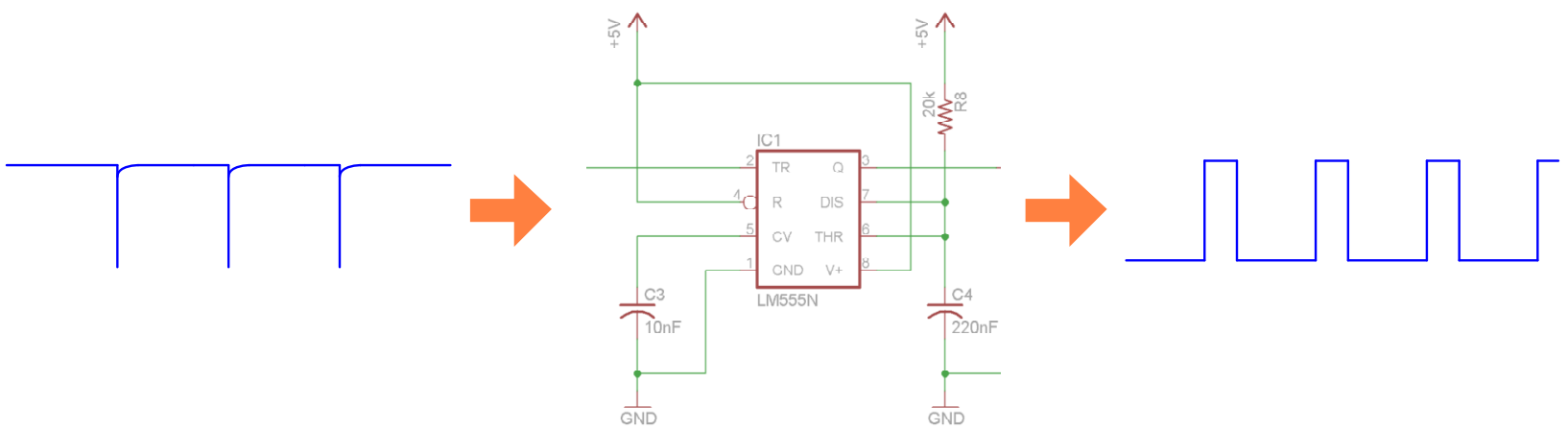

Figure 8: The pulse generator creates a pulse of a precise duration when triggered by a negative pulse. 
The Pulse Generator

The pulse generator circuit is formed around an LM555 timer chip. As shown in Figure 8, the 555 is configured as a one-shot. That is, it sends out a pulse of a precise duration every time it receives a trigger signal.

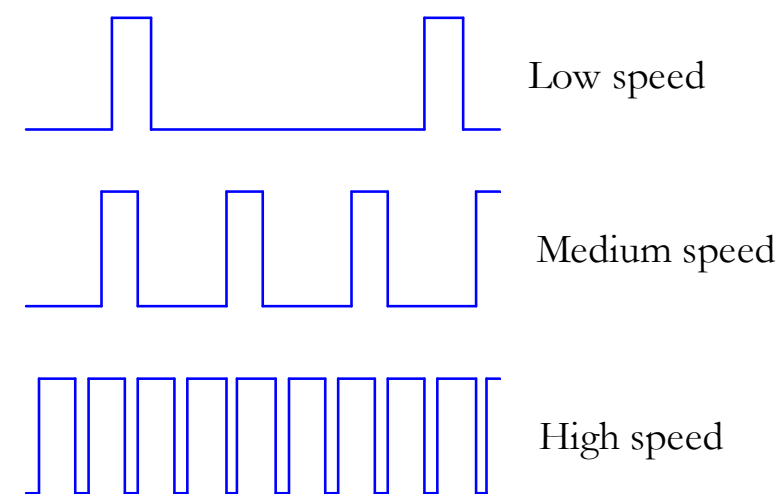

Figure 9: Output of the pulse generator for varying shaft speeds.

Figure 9 shows sample output pulse trains generated by the pulse generator for varying shaft speeds. The duration of each output pulse is determined by the values of $R_{8}$ and $C_{4}$ according to (2).

$$
T=1.1 R_{8} C_{4}
$$

Selecting a proper output pulse duration is crucial to optimal system performance. If the output pulse width is chosen to be too wide, then sufficiently quick trigger pulses from the generator (i.e. the Hall Effect being triggered by a shaft rotating faster than the tachometer circuit is designed to handle) will cause the output pulses to overlap each other and the output will remain at a constant 5V, as shown in Figure 10.

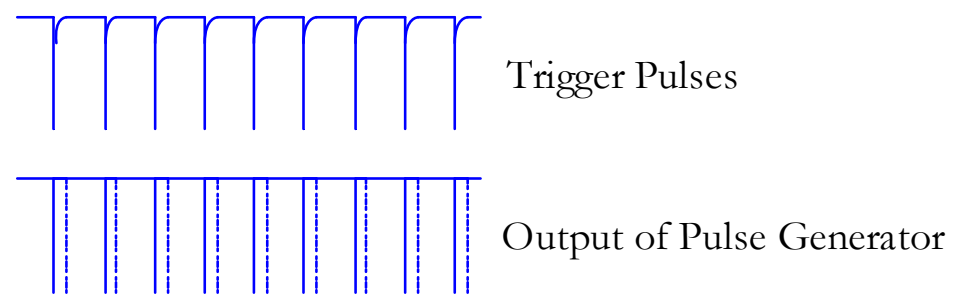

Figure 10: Saturation occurs when the time between trigger signals is less than the duration of a pulse. 
On the other hand, if the output pulses are too narrow then the resolution of the tachometer will suffer, since the average voltage of the output pulses sent to Arduino in the next stage will always be small. For the best resolution, the maximum expected speed should correspond to $5 \mathrm{~V}$ at the output, and the minimum speed should be somewhere around $0 \mathrm{~V}$. Thus, the pulses coming out of the 555 should be approximately equal to the time between pulses from the daisywheel at maximum speed.

To calculate the pulse width from the daisy wheel at maximum speed, let the number of slots in the daisywheel be $N_{S}$, and the maximum speed of the rotating shaft (in revolutions per minute) be $n_{\max }$. Then the time between pulses from the daisywheel is

$$
T=\frac{60}{n_{\max } N_{s}}
$$

For example, if the shaft is rotating at 2000rpm and there are 6 slots in the daisywheel, then the time between pulses is $5 \mathrm{~ms}$. By setting the maximum daisy wheel trigger pulse and the 555 timer output pulse widths equal, we can combine equations (2) and (3) to determine $R_{8}$ and $C_{4}$. It is much easier to keep the capacitor value constant (since there is a much smaller variety of capacitor values) and change the resistor to achieve the desired pulse length. The combined equation for the proper resistor value is

$$
R_{8}=\frac{60}{1.1 n_{\max } N_{S} C_{4}}
$$

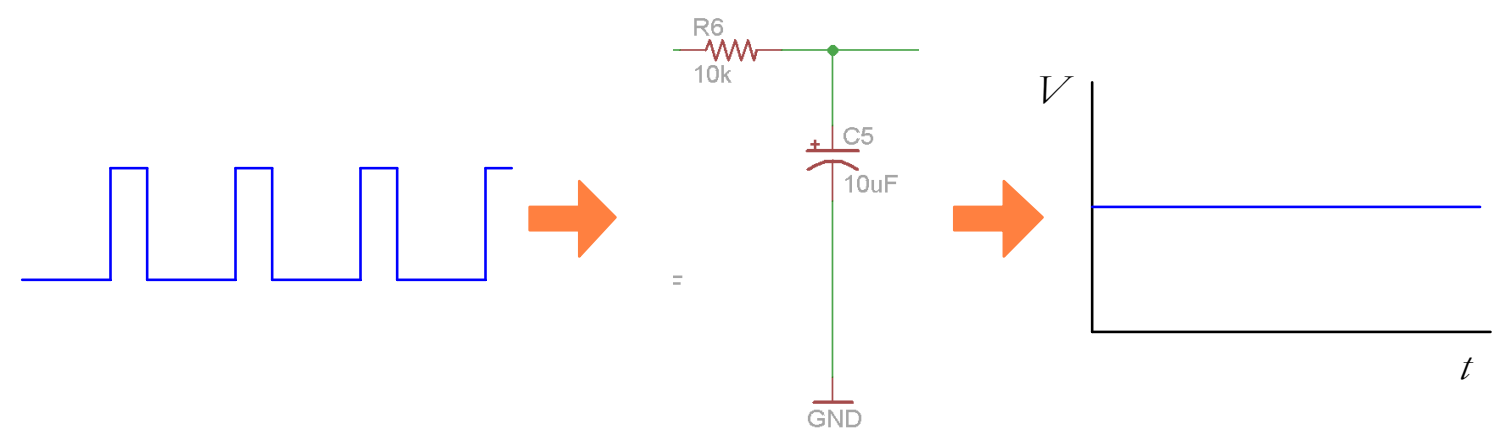

Figure 11: The lowpass filter produces the average value of the input voltage.

\section{The Lowpass Filter}

The final stage in the circuit is the lowpass filter, shown in Figure 11. The lowpass filter passes along the average value of the voltage coming into it. If the pulses are close together, the average voltage is high, and if the pulses are widely spaced, the average value is low. The lowpass filter has a cutoff frequency given by 


$$
f_{c}=\frac{1}{2 \pi R_{6} C_{5}}
$$

As with everything electronic, this circuit design is a tradeoff. If we make the cutoff frequency very low, it will make the output signal nice and smooth, but will be very sluggish in responding to changes in speed. If we make it very high, the choppy nature of the pulses will be passed through, and it will be hard for Arduino to measure the speed correctly.

During initial testing a first order filter appeared to be sufficient, however after integration into the hybrid test setup we have "upgraded" to a second order filter by copying the circuit in Figure 11. On the hybrid test setup the second order filter provided a more stable analog voltage without greatly affecting response time. Currently the second order filter is breadboarded by students but will be included in the next revision of the tachometer PCB.

For the Tachometer project, our students breadboard the circuit described above, and calculate proper values for $\mathrm{R}_{8}$ and $\mathrm{R}_{6}$. After testing the breadboarded circuits to ensure that they function at the anticipated speeds of the benchtop hybrid (roughly 0-2000rpm) the students solder the circuits onto a provided printed circuit board. The pedagogical goals of the project are simple circuit design and analysis, and electronics prototyping and troubleshooting. So far, we have found the analog tachometer to be the most reliable and "student-proof" of the designs we have tried. A full set of CAD files for the tachometer circuit PCB can be found on the project website, www.benchtophybrid.com.

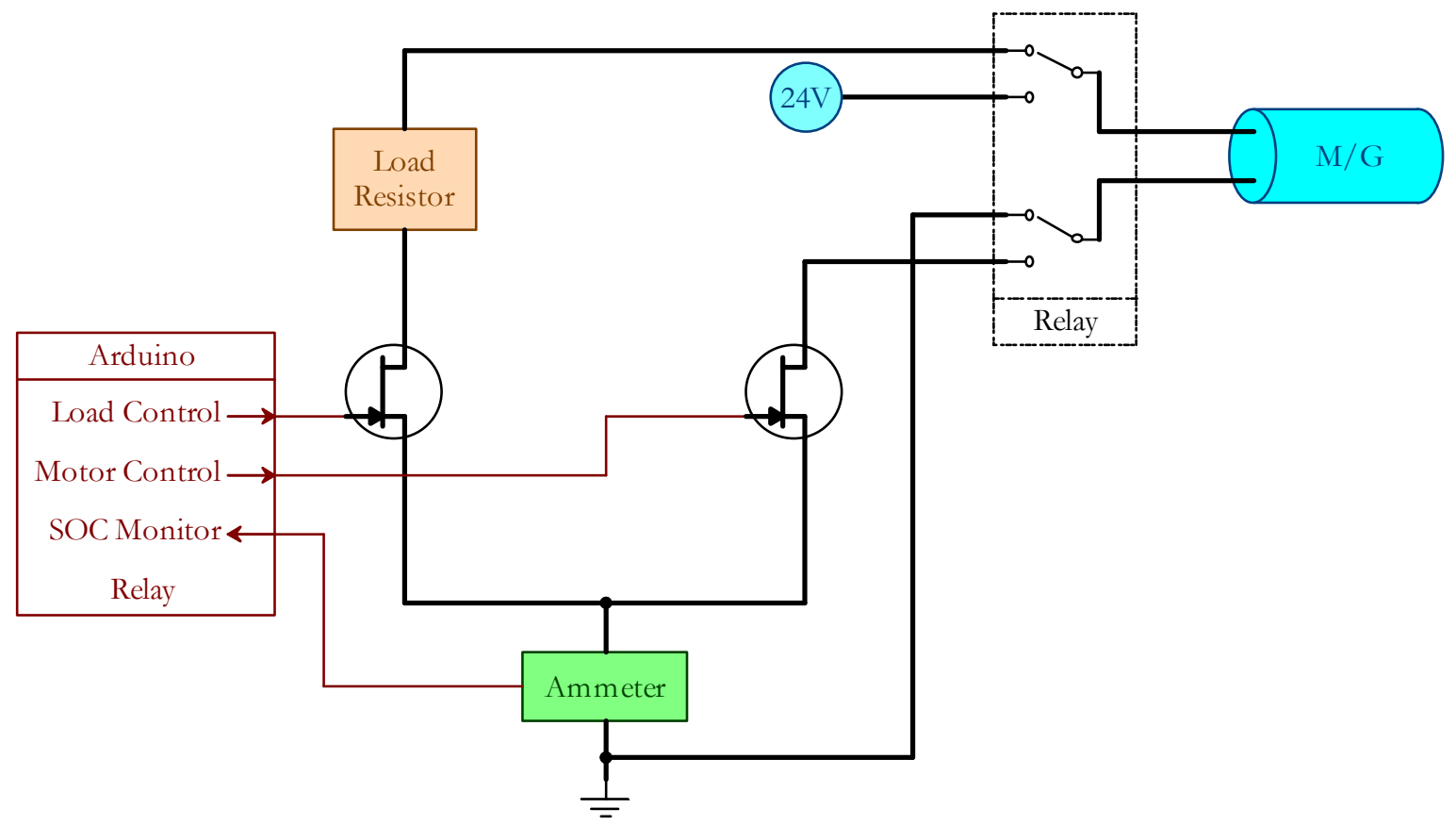

Figure 12: Functional diagram of the Motor/Generator Board. These boards are provided to the students, who use Arduino to monitor and control them. Three Motor/Generator Boards are required for each Benchtop Hybrid setup. 


\section{The Motor/Generator Board}

Figure 12 shows a functional diagram of the Motor/Generator Board. The setting of a DPDT relay determines whether the board is in "Drive" or "Generator" mode. In drive mode, a pulsewidth modulated signal from Arduino regulates the current flowing through two MOSFET transistors (IRF520) to the drive the motor. The Arduino board is protected from the rest of the circuit with an optoisolator (Lite-On LTV-827). In generator mode, the leads of the motor are connected across a bank of three $10 \Omega, 100 \mathrm{~W}$ power resistors (Riedon PF2472-10RF1). The current flowing through the load resistors is also pulse-width modulated using an IRF520 MOSFET, allowing for variable loading to simulate different hill gradients.

To monitor the current sent to the motor in drive mode - or dissipated through the load resistors in generator mode - a current sensor (Allegro ACS758LCB-050B) is inserted between the MOSFETs and ground. The signal from the current sensor is amplified using an inexpensive op-amp (LM358N) and sent to an analog input of Arduino. The full circuit diagram and board layout for the Motor/Generator board can be found at www.benchtophybrid.com.

One important feature of the Motor/Generator board is the "flyback diode" added across the leads of the motor, as shown in Figure 13. When power to the motor is suddenly turned off (as when switching from downhill mode to uphill mode) the coils in the motor create a sudden, highvoltage spike of reverse polarity. This voltage spike is high enough to damage or destroy the MOSFET transistor, even with an internal protecting diode, as we have experienced on numerous occasions. The diode allows the voltage spike to be dissipated harmlessly in the power supply and protects the transistor.

In the Benchtop Hybrid project we ask our students to breadboard a simple version of the motor drive circuitry, with MOSFET, flyback diode and optoisolator. Afterwards, the students use the faculty-created Motor/Generator boards while implementing their overall control scheme. We have found that the MOSFET transistors in particular are susceptible to being destroyed in student-built circuits during the rough-and-tumble of control system troubleshooting. 


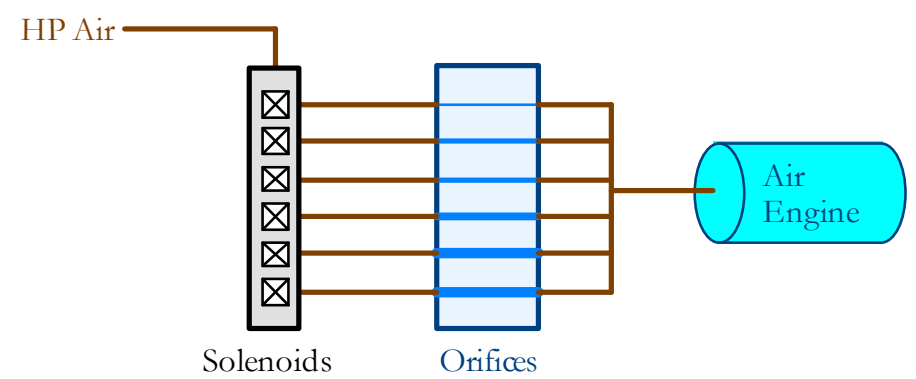

Figure 14: The air engine speed control uses six solenoids and an orifice block to regulate the flow of high-pressure air.

\section{The Solenoid Board}

In order to effect the overall control scheme for the Benchtop Hybrid, students must regulate the speed of the air engine using Arduino. In early versions of the project we used servo and stepper motor control of an air valve. Experience demonstrated that even a small opening of the air valve allowed sufficient air to pass so that the speed of the air engine was ungovernable, especially at low loads. After much trial and error, we adopted the orifice block design, shown in Figure 14, which was inspired by the fuel regulation system found on some diesel locomotives. In this system, high-pressure air is passed through a set of orifices with varying diameters; the number of open orifices is governed by the opening and closing a set of solenoid valves. Choice of orifice sizes and control logic are explained in detail in [3].

One solenoid drive unit is shown in Figure 15. The circuit is almost identical to the motor drive circuit described earlier, and there are six solenoid drive

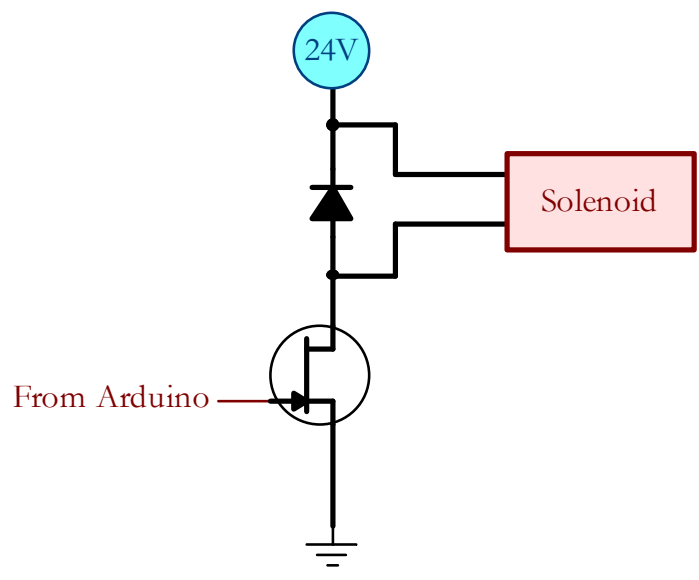

Figure 15: One of six solenoid valve drivers on the Solenoid Board. The circuit is almost identical to the motor drive circuit described earlier. units on the Solenoid Board. Provision has been made on the board for the instructor to monitor the total flow of air to the air engine as a way of measuring "fuel consumption". This is accomplished by monitoring which valves the students open and for what duration. A "fuel consumption" value is obtained using

$$
F_{\text {air }}=\int_{0}^{t} A_{1} C_{1} d t+\int_{0}^{t} A_{j} C_{j} d t+\cdots+\int_{0}^{t} A_{6} C_{6} d t
$$

Where $F_{\text {air }}$ is the total "fuel consumption", $A_{j}$ is the cross-sectional area of the orifice controlled by solenoid $j$, and $C_{j}$ is the binary control command of solenoid $j$ having a value of 0 or 1 .

\section{Conclusion}

The Benchtop Hybrid project was started in 2011, and will continue for the next two or three student cohorts. Since then, we have learned many valuable lessons in developing large-scale electromechanical projects for our students. The primary key to a successful multi-year project is 
setting achievable semester goals that are relevant to course curriculum yet challenging for students of that academic year.

As this is a multi-year, large scale design project, many of the components designed and fabricated in early semesters (e.g. the tachometer and air engine) must be reused in later semesters. For simple, robust systems such as the tachometer this poses no difficulty. For more complicated systems, such as the air engine and motor/generator board, we allow students to use "faculty models" in later semesters. This eliminates the need for troubleshooting older, studentbuilt systems so that students can concentrate on learning the current course material.

One unexpected result of our multi-year project has been the development of camaraderie between students in different academic years, since every student is involved in the same project. Cross-year communication has increased, and has also spurred friendly competition to outperform the previous cohort of students, thus driving the quality of projects up.

\section{Bibliography}

[1] Toyota Motor Corporation, "Toyota Hybrid Transmission II (THS II)," Toyota Public Affairs Division, Japan, May 2003. [Online]. Available: http://www.evworld.com/library/toyotahs2.pdf. [Accessed 20 February 2013].

[2] E. W. Constans, J. Kadlowec, K. K. Bhatia, H. Zhang, T. Merrill and B. Angelone, "Integrating the Mechanical Engineering Curriculum using a Long-Term Green Design Project: Part 1: The Hybrid Powertrain," ASEE Annual Conference, 2012.

[3] E. W. Constans, M. S. Acosta, K. K. Bhatia, H. Zhang and J. Kadlowec, "Development and Implementation of a Control Strategy for a Hybrid Power Train System in a Classroom Setting," ASEE Annual Conference, 2014.

[4] M. S. Acosta, E. W. Constans, K. K. Bhatia, J. Kadlowec, T. Merrill, H. Zhang and B. Angelone, "Integrating the Curriculum using a Bench-Scale Hybrid Power Train," SAE 2014 World Congress \& Exhibition, 2014.

[5] E. W. Constans, S. I. Ranganathan and W. Xue, "Design and Fabrication of a Planetary Gearset as Part of a Hybrid Powertrain," in ASEE Annual Conference and Exposition, Seattle, WA, 2015.

[6] K. K. Bhatia and E. W. Constans, "Novel Use of an Engine Design Project to Cross-Link Knowledge from Courses in both Mechanical Design and Thermodynamics," Frontiers in Engineering Education, 2006. 